1 Universidade Federal da Bahia (UFBA), Instituto de Saúde Coletiva (ISC) Salvador (BA), Brasil. drcrisdosoriofigueroa@ gmail.com

\section{El papel de la atención primaria en Guatemala para la Covid-19: límites y potencialidades}

\author{
Guatemala's primary healthcare role in Covid-19: limits and \\ potentialities
}

Cristian David Osorio Figueroa ${ }^{1}$

DOI: $10.1590 / 0103-1104202113016$

\begin{abstract}
RESUMEN El presente ensayo buscó describir el rol de la Atención Primaria en Salud (APS) en Guatemala en la implementación de políticas públicas para la contención de la pandemia, y discutir sus límites y potencialidades. Fueron utilizadas fuentes oficiales de gobierno, cotejadas con experiencias internacionales y literatura científica sobre APS. Inicialmente se describieron el contexto sociodemográfico y el sistema de salud guatemalteco y las principales medidas gubernamentales para contener la Covid-19. Para analizar el papel de la APS se seleccionaron cuatro líneas de análisis: abordaje de la vulnerabilidad social, coordinación del cuidado, vigilancia epidemiológica y promoción e intersectorialidad en salud. Se discuten las dificultades históricas del sistema de salud guatemalteco, que repercuten en la gestión de la pandemia con enfoque hospitalario, inversión temporal, medidas flexibles de aislamiento social y ausencia de diálogo con la población. La experiencia internacional indica que la APS territorializada, con adscripción poblacional, tiene impacto en la disminución de las inequidades, en la coordinación del cuidado para promoción de la salud, en el fortalecimiento de los sistemas de vigilancia epidemiológica y en el incremento de la intersectorialidad. Guatemala debe reconocer las potencialidades de la APS para realizar reformas estructurales y organizacionales con perspectiva intercultural, de género, participación social e intersectorialidad.
\end{abstract}

PALABRAS-CLAVE Atención Primaria de Salud. Infecciones por coronavirus. Guatemala. Pandemias.

ABSTRACT The present essay sought to describe the role of Primary Healthcare (PHC) in Guatemala, discussing its limits and potentialities at public policies implementation to contain the Covid-19 pandemic. Official government sources were compared to international experiences and scientific literature on PHC. A socio-demographic characterization and health system description were made, followed by stating the government's measures to contain the pandemic. It proposes four lines to analyze the role of PHC: addressing social vulnerability, care coordination, epidemiological surveillance, and health promotion and intersectionality. It discusses the historical deficiencies of the Guatemalan health system affecting the pandemic's management, characterized by a hospital-centered approach, temporary investment, flexible social distancing strategies, and the lack of dialogue with the population. International experience indicates that territorialized PHC with population assignment has an impact on reducing inequities, coordinating care for health promotion, strengthening epidemiological surveillance systems, and increasing intersectionality. Guatemala must recognize the potential of PHC to carry out structural and organizational reforms with intercultural, gender equity, social, and intersectional perspectives.

KEYWORDS Primary Health Care. Coronavirus infections. Guatemala. Pandemics. 


\section{Introducción}

El 11 de marzo del 2020, la Organización Mundial de la Salud (OMS) declara pandemia la enfermedad por coronavirus 2019 (Covid19), cuyo agente etiológico es el coronavirus del síndrome respiratorio agudo severo 2 (Sars-CoV2), la cual puede presentarse con amplia variabilidad clínica desde una forma asintomática hasta una forma grave que requiere soporte ventilatorio $\mathbf{1}$. Actualmente se considera que el $40 \%$ posee síntomas leves, $40 \%$ moderados, $15 \%$ graves y $5 \%$ pueden avanzar a un estado crítico ${ }^{2}$. Al encontrarse aún sin tratamiento definido o vacuna y dada su alta transmisibilidad, las políticas para su enfrentamiento se basaron, aunque con diferentes matices, en estrategias de aislamiento físico, fortalecimiento de los sistemas de salud, programas de protección social para grupos vulnerables y políticas para la contención de consecuencias económicas derivadas de las medidas tomadas ${ }^{\mathbf{3}, 4}$.

El aporte de la determinación social de la salud permite incluir en la matriz analítica las dimensiones de orden política, social, económica, cultural y ecológica, y cuestiona los supuestos que generalizan o disminuyen la relevancia de procesos históricos, contextos, territorios, colectivos y sujetos en el entendimiento de fenómenos como pobreza y enfermedad ${ }^{5}$. Siendo esto necesario para comprender la respuesta del sistema de salud más allá de la expresión individual de las enfermedades.

Ampliar la comprensión del problema desde un pensamiento complejo genera nuevas respuestas de construcción participativa, transdisciplinaria e intercultural y en relación con el desarrollo económico y sociocultural del país ${ }^{6,7}$. Por ello, la Atención Primaria en Salud (APS), en su definición amplia, se presenta como un eje de análisis ideal sobre el abordaje de la pandemia.

Desde la firma de la declaración de Alma Ata, la estrategia de la APS es sugerida como el camino adecuado para el fortalecimiento de los sistemas sanitarios, la reducción de las inequidades y la incidencia sobre la determinación de la salud ${ }^{8}$. La estrategia se destaca dado que la pandemia ha exacerbado la condición de vulnerabilidad en América Latina, especialmente de pueblos originarios, mujeres, personas en situación de calle, poblaciones con discapacidad, moradores de asentamientos urbanos precarios, personas en trabajo informal y de la tercera edad ${ }^{9}$.

La pandemia puso bajo presión a los sistemas de salud, su capacidad instalada de camas hospitalarias, acceso a unidades de cuidado intensivo y ventiladores ${ }^{10}$. Al mismo tiempo, otros retos fueron impuestos como la manutención de oferta regular de atención, sistemas de vigilancia para detección de casos en las comunidades y población en condición de vulnerabilidad exacerbada por las medidas de aislamiento social. De hecho, el fracaso de la gestión centrada en las medidas hospitalocéntricas requirió su modificación a un enfoque comunitario"1, para lo cual, la reorganización de las actividades de la APS alrededor del mundo ha sido fundamental para una respuesta efectiva ${ }^{\mathbf{1 2}}$.

El presente ensayo tiene por objetivo describir las principales políticas públicas en Guatemala para el combate a la pandemia por Sars-CoV2 e identificar el papel otorgado a la APS. Para ello fueron seleccionados cuatro ejes de análisis: abordaje de la vulnerabilidad social, coordinación del cuidado, vigilancia epidemiológica y promoción e intersectorialidad en salud, apuntando límites y potencialidades. Para la realización del texto se hizo énfasis en fuentes oficiales del gobierno. Aquellas medidas no publicadas en documentos oficiales se obtuvieron de fuentes periodísticas e informes de la sociedad civil. Inicialmente se exhibe el contexto sociodemográfico guatemalteco y su sistema de salud, para posteriormente presentar cada uno de los ejes de análisis seleccionados, cotejando con la literatura especializada en APS y experiencias internacionales, y concluir presentando las consideraciones finales. 


\section{Contexto sociodemográfico guatemalteco}

Guatemala es el país más poblado de América Central. Su población se encuentra conformada por cuatro pueblos: maya, xinca, garífuna y mestizo. Entre los cuales, los mayas representan el $41 \%$ de la población divididos en 22 comunidades sociolingüísticas ${ }^{13}$ lo que le confiere al país un carácter multilingüe con 25 idiomas en total.

Aunque posee el mayor crecimiento económico de la región centroamericana, su riqueza lingüística y cultural no se refleja de forma equitativa, de hecho, es uno de los países más desiguales de América Latina. Estas desigualdades se profundizan en áreas rurales y entre mujeres mayas, en un contexto de falta de acceso a servicios de salud, a seguridad alimentaria, educación, empleo digno, vivienda y servicios básicos; de hecho, el 60\% de la población guatemalteca es multidimensionalmente pobre y esto aumenta a un $80 \%$ en comunidades mayas ${ }^{\mathbf{1 4}}$.

La experiencia del país en incidir sobre la determinación social de la salud no ha sido prioridad desde el punto de vista del gasto social. De hecho, es el segundo más bajo de América Latina con tan sólo el 7\% del PIB y un promedio per cápita destinado a programas sociales equivalente a 230 dólares. De los cuales, el $42.3 \%$ se destina a educación, $18.2 \%$ a programas de protección social y $15.3 \%$ a salud. El resto se distribuye en vivienda y servicios comunitarios, recreación y medio ambiente ${ }^{15}$. Otras características sociodemográficas se resumen en la tabla $1^{13,16-23}$.

Tabla 1. Variables sociodemográficas, sanitarias y nutricionales de Guatemala

\begin{tabular}{|c|c|c|c|}
\hline Dimensión & Variables & 2015 & 2018 \\
\hline \multirow[t]{5}{*}{ Demografía } & Población total (millones) & $12.2(2002)$ & 14.9 \\
\hline & Población 15-64 años (\%) & 52.8 & 61.0 \\
\hline & Población maya (\%) & s.d $d^{*}$ & 41.7 \\
\hline & Población mestiza (\%) & $59.0^{*}$ & 56.0 \\
\hline & Tasa de fecundidad (hijos por mujer) & $3.4(2010)$ & 3.8 \\
\hline \multirow[t]{2}{*}{ Educación } & $\begin{array}{l}\text { Tasa ajustada de matrícula neta, primaria ( } \% \text { de } \\
\text { niños en edad de escuela primaria) }\end{array}$ & 87.8 & 89.2 \\
\hline & $\begin{array}{l}\text { Persistence to last grade of primary, total (\% of } \\
\text { cohort) - Guatemala }\end{array}$ & $78.3(2016)$ & 71.8 \\
\hline Empleo & Población en trabajo informal (\%) & $77(2013)$ & \\
\hline \multirow[t]{9}{*}{ Salud } & Tasa de mortalidad infantil (por cada 1000) & 23.7 & 21.4 \\
\hline & $\begin{array}{l}\text { Tasa de mortalidad, menores de } 5 \text { años (por cada } \\
\text { 1000) }\end{array}$ & 28.3 & 25.4 \\
\hline & $\begin{array}{l}\text { Razón de mortalidad materna (por cada 100000) } \\
\text { (indígena; no indígena) }\end{array}$ & $79.3 ; 138.9$ & sin dato \\
\hline & Gasto en salud en \% del PIB & 5.9 & 5.7 \\
\hline & Gasto de bolsillo (\% de los gastos actuales en salud) & 55.7 & 57.5 \\
\hline & $\begin{array}{l}\text { Gasto gubernamental (\% de los gastos actuales en } \\
\text { salud; \% del PIB) }\end{array}$ & $37.9 ; 2$ & $36.0 ; 2$ \\
\hline & Camas hospitalarias (por cada 1000) & 0.58 & $0.44(2017)$ \\
\hline & Profesionales de medicina (por cada 1000) & $0.904(2009)$ & 0.355 \\
\hline & Personal de enfermería (por cada 1000) & 0.054 & 0.074 \\
\hline
\end{tabular}


Tabla 1. (cont.)

\begin{tabular}{llrr}
\hline Dimensión & Variables & $\mathbf{2 0 1 5}$ & $\mathbf{2 0 1 8}$ \\
\hline Nutrición & Inseguridad alimentaria grave (\%) & 14.3 (2014) & 16.4 \\
& Inseguridad alimentaria moderada a grave (\%) & s.d. & 43.6 \\
Pobreza multidimen- & Índice de pobreza multidimensional (\% general; \% & s.d. & 60\%; 80\% \\
sional & maya) & 0,627; medio & $0.651 ;$ medio \\
Desarrollo humano & Índice de Desarrollo Humano (índice; clasificación) & 0.443 & 0.472 \\
& Índice de Desarrollo Humano ajustado por desigual- & & 48.3 \\
& dad & 48.3 & $0.492 ; 118$ \\
\hline
\end{tabular}

Fuente: Elaboración propia $\mathbf{1 3}^{\mathbf{1 6}-\mathbf{2 3}}$.

*Censo 2002 realizó clasificación en indígena y no indígena sin desagregación por pueblo.

\section{Caracterización del sistema de salud guatemalteco}

El sistema de salud de Guatemala se caracteriza por ser altamente segmentado (cuadro

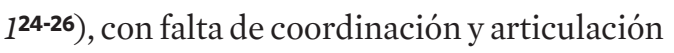
en la prestación de servicios de salud por las distintas instituciones del Estado y el sector privado. El sector público incluye, primero, al Ministerio de Salud Pública y Asistencia Social (MSPAS), el cual brinda atención al $70 \%$ de la población por medio de tres niveles de atención ${ }^{24}$. En segundo lugar, el Instituto Guatemalteco del Seguro Social (IGSS) con una cobertura del $20 \%$ de la población, principalmente urbana y del sector de servicios ${ }^{27}$.

Cuadro 1. Caracterización del sistema de salud guatemalteco por subsector

\begin{tabular}{|c|c|c|c|}
\hline Subsector & Prestador de servicios & Población usuaria & Financiamiento \\
\hline \multirow[t]{4}{*}{$\begin{array}{l}\text { Subsector } \\
\text { público }\end{array}$} & $\begin{array}{l}\text { Ministerio de Salud Pública y Asis- } \\
\text { tencia Social (MSPAS) }\end{array}$ & $\begin{array}{l}\text { Población general (usualmente po- } \\
\text { blación en situación de vulnerabilidad } \\
\text { social) }\end{array}$ & $\begin{array}{l}\text { Impuestos, préstamos } \\
\text { o donaciones }\end{array}$ \\
\hline & $\begin{array}{l}\text { Instituto Guatemalteco de Seguri- } \\
\text { dad Social (IGSS) }\end{array}$ & Población afiliada (trabajo formal) & $\begin{array}{l}\text { Contribuciones patro- } \\
\text { nales y de afiliados }\end{array}$ \\
\hline & Hospitales militares y policiales & Fuerzas de seguridad & Presupuesto asignado \\
\hline & Municipalidades & Población local & Presupuesto municipal \\
\hline \multirow[t]{2}{*}{$\begin{array}{l}\text { Subsector } \\
\text { privado }\end{array}$} & Privados no lucrativos & $\begin{array}{l}\text { Población general (usualmente po- } \\
\text { blación en situación de vulnerabilidad } \\
\text { social) }\end{array}$ & $\begin{array}{l}\text { Cooperación externa, } \\
\text { donaciones }\end{array}$ \\
\hline & Privados lucrativos & Población con capacidad de pago & $\begin{array}{l}\text { Pago directo de bolsillo, } \\
\text { aseguramiento privado }\end{array}$ \\
\hline \multirow[t]{3}{*}{$\begin{array}{l}\text { Subsector } \\
\text { comunitario }\end{array}$} & Modelo de medicina Maya & Población abierta & $\begin{array}{l}\text { Pago directo de bolsillo } \\
\text { o en especie }\end{array}$ \\
\hline & Otros modelos alternativos & Población abierta & $\begin{array}{l}\text { Pago directo de bolsillo } \\
\text { o en especie }\end{array}$ \\
\hline & Modelo de autoatención & Familiares & Sin pago \\
\hline
\end{tabular}

Fuente: Elaboración propia24-26. 
La organización de la atención evidencia una alta fragmentación institucional, con la existencia de 20 programas verticales focalizados en enfermedades, riesgos, poblaciones específicas, cuidados episódicos agudos y atención hospitalaria, con ausencia de un modelo de atención integral, continuo e integrado ${ }^{28}$.

El gasto público en salud representa el 1.7\% del PIB, y a pesar del contexto de pobreza, el $57.5 \%$ del gasto de salud proviene de bolsillo ${ }^{15}$, pudiendo repercutir en gastos catastróficos para las familias. La falta de inversión proviene de la reforma de ajuste estructural de los años 90. Durante ese periodo se inició la tercerización de la atención a organizaciones no gubernamentales por medio del Programa de Extensión de Cobertura que consistió en una modalidad de APS selectiva, que prestaba atención por paquetes básicos de servicios en jornadas médicas descontinuas ${ }^{29}$. Al mismo tiempo, no ha existido inversión en talento humano ni en el fortalecimiento de la red de atención ${ }^{\mathbf{2 8}}$.

Respecto a la atención primaria, se calcula que posee una brecha de 6,000 puestos de salud y 2,000 centros de salud, con problemas recurrentes como ausencia de medicamentos, débil capacidad resolutiva y ausencia de una red de cuidados intermedios para atención ambulatoria ${ }^{25}$. En los puestos de salud, la atención en el área rural es llevada a cabo por personal técnico de enfermería con formación de un año, sin apoyo en red y sin la debida valorización. La disponibilidad de recursos humanos es escasa y no responde a las necesidades identificadas en el perfil epidemiológico y demográfico del país, presentando alta concentración en áreas urbanas ${ }^{\mathbf{3 0}}$.

La APS se ve debilitada en términos de financiamiento, debido a la preeminencia presupuestaria del sector hospitalario, el cual ocupa el $60 \%$ del presupuesto del Ministerio de Salud. Adicionalmente, existen inequidades en la distribución de los recursos ya que ante los 44 hospitales que posee el país, el 36\% del presupuesto hospitalario se destina únicamente a dos centros ubicados en la capital ${ }^{31,32}$. Como consecuencia, los centros hospitalarios ubicados en otros departamentos del país, poseen una baja capacidad de resolución por ausencia de especialistas, cuidados intensivos y tecnología diagnóstica, una limitada capacidad instalada de camas ( 0.6 camas por cada 1,000 habitantes ${ }^{33}$ ) y menos de 300 unidades de cuidados intensivos ${ }^{9}$ a nivel nacional.

En el 2017, se realizó el único ejercicio de crear territorios para establecer zonas de adscripción poblacional. Para ello se dividió el país en sectores conformados por comunidades $\mathrm{y}$ familias, y fueron proyectados un total de $2,679^{34}$. Sin embargo, no se dio continuidad al fortalecimiento de la infraestructura o asignación de equipos para la atención ${ }^{35}$, regresando nuevamente a la prestación de atención por paquetes básicos.

Estudios de antropología en salud 25,36,37 caracterizan en el ámbito comunitario los sistemas de salud maya, garífunas, xincas y populares (en referencia al pueblo mestizo), con amplia presencia en el territorio nacional y modalidades de atención colectivas, familiares e individuales.

\section{Medidas sociales y económicas en respuesta a la pandemia por Covid-19 en Guatemala}

El 13 de marzo de 2020 se confirmó el primer caso en Guatemala por el Sars-CoV2 y desde entonces se comenzaron a implementar una serie de medidas de contención parecidas a las realizadas por otros países. Entre ellas, la restricción de la interacción social por medio de suspensión de actividades sociales, clases en todos los niveles educativos, eventos religiosos y públicos y ferias patronales. También se suspendió el transporte público tanto urbano como extraurbano, que conecta los diferentes municipios del país. Entre las excepciones se encontraban los sectores de salud, alimentario, de seguridad, energético, telecomunicaciones, comercios y servicios básicos. Sin 
un instrumento legal de amparo se instó a que quienes pudieran trabajar en sus casas de manera virtual lo hicieran ${ }^{38}$. Posteriormente, las medidas serían flexibilizadas.

Debido a la presión ejercida por la pandemia, a las recomendaciones internaciona${ }^{l e s^{39}}$ y a la sociedad civil organizada, fueron aprobados en el Congreso de Guatemala, diez programas y su respectivo financiamiento por medio de préstamos, con el objetivo de cubrir a las poblaciones en condición de vulnerabilidad. Estos básicamente consisten en suspensión de contratos laborales, bono único al comercio popular (US\$130), fondo de crédito de capital de trabajo para empresas afectadas por la crisis, bono salarial para el personal de salud, subsidio parcial al pago de energía eléctrica, ampliación del programa de aporte económico al adulto mayor, bono familiar (US\$130 mensuales por tres meses), dos programas de ayuda alimentaria directa (uno proveniente de donación internacional) y el programa de alimentación escolar que otorga una cesta alimentaria con un valor aproximado a US $\$ 7.30^{40}$.

La formulación de políticas públicas ocupó el tiempo-espacio de las vidas humanas, con fuertes impactos en las prácticas de salud diferenciadas sobre las personas, en particular aquellos grupos vulnerables. Funcionaron así, como dispositivos biopolíticos centrados en los cuerpos y únicamente en el virus ignorando todo el contexto, la interculturalidad y la perspectiva de género 5 .

Por ejemplo, las medidas de restricción de transporte público trajeron gastos catastróficos como consecuencia de la necesidad de contratación de transporte privado para la movilización, aumento de precios en los alimentos, atraso de ayuda humanitaria y largas caminatas para comprar en los mercados autorizados ${ }^{41}$.

Además, el flujo de migración interna, principalmente de mujeres indígenas al sector de maquilas, se vio afectado por despidos y, ante la falta de protección social del trabajo doméstico, este pasó a darse en situación de reclusión lo cual implica largas jornadas laborales sin mejora salarial y oportunidad de recreación ${ }^{\mathbf{4 2}}$. Por su parte, la población trans cuyas fuentes de ingresos provienen de negocios informales o de trabajo sexual, ambos paralizados, se vio sin ninguna política de protección social específica ${ }^{43}$.

Durante el periodo de confinamiento la violencia intrafamiliar se agravó $\mathbf{4 4}$ y el número de embarazos en niñas y adolescentes se duplicó en comparación con el año $2019^{45}$, mientras que la desnutrición y la inseguridad alimentaria se triplicaron ${ }^{\mathbf{4 1}}$. Las brechas de género en el cuidado reproductivo han aumentado, debido al trabajo doméstico y educativo, y dejando a las mujeres con el rol de velar por la contención del contagio ${ }^{44}$. Además, la proliferación de permisos otorgados a empresas para continuar su funcionamiento con jornadas laborales reducidas permitió la proliferación de personas en las calles y tráfico como en cualquier día antes de la pandemia9 .

\section{Medidas para el fortalecimiento de la atención en el contexto por Covid-19 en Guatemala}

En Guatemala, la estrategia adoptada por el gobierno para aumentar la capacidad resolutiva del sistema de salud fue la creación de un hospital de campaña con capacidad de 3000 camas, que sería el mayor de Centroamérica y en la práctica fue montado por donaciones del sector privado. Este hospital sería dotado de talento humano para la atención de casos moderados y con camas de cuidados intensivos. $\mathrm{Al}$ comenzar su funcionamiento, la ausencia de planificación fue desnudada, no contaba con aspectos logísticos para la alimentación, limpieza y servicios básicos de higiene y agua potable para las personas internadas ${ }^{\mathbf{4 6}}$.

Aunque los países optaron por fortalecer sus sistemas públicos de salud con valorización de la APS, en Guatemala se aprobó el Programa 
Seguro Médico Escolar para estudiantes de nivel pre-primaria y primaria en centros educativos públicos, con un costo de 200 millones de quetzales ${ }^{47}$ para atención médica y farmacéutica por enfermedad común y accidente. Además, se implementaron kits de medicamentos para pacientes leves sin protocolos oficiales de monitoreo remoto, como parte de la estrategia para la APS ${ }^{48}$, con medicamentos de limitada evidencia como la ivermectina ${ }^{49}$. La ejecución presupuestaria de los recursos que serían destinados para el fortalecimiento de la red de atención fue del $4 \%{ }^{\mathbf{5 0}}$.

En el contexto guatemalteco el sistema de vigilancia epidemiológica es débil dado su corto alcance y el sistema de información no permite tomar decisiones pues su orientación es hacia la producción. La capacidad de testeo ha sido limitada y centralizada en grandes centros urbanos con lo cual la magnitud del flujo de circulación del virus es desconocida. La abertura de las actividades comerciales y económicas debería acompañarse de una vigilancia activa de casos.

No existen programas para investigación y desarrollo. De hecho, el valor dado a los números en detrimento del valor dado a la vida coloca uno de los cuadros más perversos, estableciendo metas que se reducen a la productividad, sin mecanismos de impacto sobre el proceso de atención y sus efectos en términos de acceso, equidad e integralidad51.

\section{Atención Primaria en Salud y vulnerabilidad social}

Ante los efectos de las políticas implementadas, diferenciados según población y territorio, el potencial de la APS sería fundamental, debido al conocimiento de las comunidades, familias e individuos para buscar alternativas y soluciones intersectoriales para reducir las consecuencias. Existe suficiente evidencia para decir que sistemas de salud con APS fuerte, poseen menores inequidades en salud, mayor conocimiento de su población y territorio así como de los problemas a los cuales se enfren$\tan ^{52}$, y consecuentemente pueden funcionar en sinergia con otros sectores para potenciar mejores condiciones de vida de forma participativa y emancipatoria ${ }^{7}$.

De hecho, la APS se estableció como una estrategia fundamental de la pandemia por organismos internacionales a raíz de las experiencias en diversos países europeos. La responsabilidad por la coordinación y la continuidad de la atención, la ampliación de la vigilancia epidemiológica, la interrupción de cadenas de transmisión del virus, la protección de grupos vulnerables y la oferta de soporte emocional y psicosocial fueron atribuciones de la APS durante la pandemia ${ }^{53}$.

Dentro de las cinco respuestas que formaron parte del plan para la atención primaria en Australia, una de ellas se enfocó específicamente en la protección de población vulnerable, por medio de su identificación, rastreo y seguimiento. Entre ella estaban la población indígena, personas de la tercera edad y población en situación de calle ${ }^{54}$. Mientras tanto otros países readaptaron sus actividades para dar continuidad al seguimiento de su población adscrita y reforzar cuidados, con desafíos impuestos por la alta demanda y las desigualdades al acceso a la tecnología 55 .

La organización de las prácticas y acciones en salud desde la perspectiva de la integralidad y territorialización del cuidado es fundamental para lograr la articulación de acciones intersectoriales de base local ${ }^{56}$, considerando la diversidad de contextos y vulnerabilidades. El uso del territorio permite abordar las interacciones humanas-ecológicas desde una perspectiva positiva de la salud, ya que históricamente el territorio ha sido relegado a un espacio exclusivo para la atención a la enfermedad57, sin embargo, al ampliar el propio concepto también se impacta el campo de posibilidades de intervención de la APS.

Existen varias limitaciones para una atención integral, territorializada y con población adscrita en Guatemala; principalmente su baja cobertura y ausencia de mecanismos 
de adscripción poblacional. De ese modo las tareas de pensar en la territorialización como eje organizador e integrador de prácticas de salud que promuevan el actuar intersectorial, la participación social y la adecuación a las características socioculturales y sanitarias, con identificación de las poblaciones vulnerables para su protección; y de garantizar el acceso a una atención integrada e integral, se hacen difíciles en un contexto de baja accesibilidad y desfinanciamiento histórico de la APS en Guatemala.

\section{Coordinación del cuidado por la APS}

Ante la visibilidad mediática sobre medidas de aislamiento físico y las estadísticas sobre el número de casos, mortalidad, letalidad y número de camas de cuidados intensivos, otras estrategias adoptadas por los sistemas sanitarios quedaron invisibilizadas. Por ejemplo, experiencias exitosas tanto en países europeos $^{53}$ como en América Latina ${ }^{58}$, muestran que el papel de la APS en la coordinación, vigilancia, gestión del riesgo y conexión con mecanismos de gobernanza regionales, fueron fundamentales para una respuesta adecuada.

La coordinación del cuidado y la integración de la red de servicios de salud ya era apuntada como una necesidad $\mathbf{5 9}$ para países que lograron responder mejor al contexto pandémico, como Nueva Zelanda. La APS cumplió un papel importante de protección inclusive para la atención de casos asintomáticos, visitas domiciliares y seguimiento de $\operatorname{casos}^{55}$, garantizando así la capacidad resolutiva a nivel local para evitar al máximo el desplazamiento hacia hospitales, lo cual es fundamental dada la alta transmisibilidad del virus. Evitar la pérdida de la continuidad de la atención y mantener actividades esenciales fue colocado como otro elemento fundamental ${ }^{53}$ para la estrategia de atención primaria.

En esos países, el fortalecimiento de la APS fue una prioridad puesta en marcha, inclusive junto a mecanismos de coordinación única entre público y privado, aumento expresivo del gasto en salud y eliminación de copagos ${ }^{53}$. La lógica de la reducción del gasto y de la racionalización de los servicios de salud provocada por la crisis del $2008^{60}$, muestra que en los países de la Organisation for Economic Co-operation and Development (OECD) en un escenario pre-pandemia ya se veía poca eficiencia, baja e inefectiva capacidad de respuesta e inequidad en el acceso a la atención primaria ${ }^{52}$, lo cual requirió mecanismos de reversión en el contexto pandémico.

Italia, donde el efecto del Covid fue dramático ${ }^{61}$, durante 2010-2019, comenzó un proceso de privatización progresivo con pérdidas substantivas de financiamiento y personal de salud'62. España, un sistema de salud bien evaluado, venía con una década de austeridad que redujo el talento humano y la capacidad del sistema de salud. Inclusive la ausencia de mecanismos de coordinación y la débil vigilancia epidemiológica fueron apuntados como causales de la falta de preparación española para atender las demandas de la pandemia, junto al desfinanciamiento ${ }^{63}$.

Estudios en Brasil con otras patologías ${ }^{64}$ muestran que un factor que afecta la búsqueda de servicios de salud es la condición socioeconómica. Considerando el contexto de Guatemala y los niveles de pobreza y falta de acceso a servicios de salud, una de cada 4 personas prefiere automedicarse ${ }^{65}$, lo cual podría aumentar la subnotificación, registrándose solamente los casos de gravedad que llegan a centros hospitalarios. La ausencia de servicios ambulatorios, el cierre de las consultas externas y una baja capacidad de respuesta de urgencias, representan un riesgo para el seguimiento de casos crónicos y sus consecuentes complicaciones en el contexto pandémico. Con todo esto, se perciben obstáculos estructurales y coyunturales para consolidar la APS como coordinadora y ordenadora del cuidado.

Por su parte, contrario a las recomendacio$n^{6} s^{66}$, el papel de la APS guatemalteca no se encuentra en protocolos oficiales, quedando 
así sin oportunidad de presentarse como una estrategia eficaz para la coordinación de la atención, la cual fue dirigida a los hospitales. La gestión del talento humano no se acompañó de un mapeo de disponibilidad, necesidades y brechas para ser completadas, sea por mecanismos de reclutamiento o de reorganización. La contratación ha sido irregular e inclusive algunos están ejerciendo labores sin contratación ni remuneración. La falta de dotación de equipos de protección personal ha sido un factor de riesgo tanto para el personal hospitalario como para el de la atención primaria, incluso provocando la muerte de personal de salud, aunque declaraciones gubernamentales atribuyan estas muertes a relajar las medidas fuera del horario laboral67.

\section{Atención Primaria en Salud y vigilancia epidemiológica}

Un programa de vigilancia para la toma de decisiones por medio de un flujo de información donde sea la APS la involucrada, puede ser útil para la reducción de nuevas infecciones en las comunidades $^{68}$. Además de notificar y detectar nuevos casos, es importante el acompañamiento y el aislamiento de casos y contactos ${ }^{11}$, cuidando y considerando las vulnerabilidades de dichas personas. Sin embargo, la capacidad de testeo en Guatemala ha sido limitada.

El potencial de los agentes comunitarios en salud por su capacidad de interlocución y transmisión de mensajes informativos, claros y concisos, ha sido identificado"1. Esto, considerado en el contexto guatemalteco bajo otras modalidades de acuerdo con su modelo de atención, tendría un potencial enorme dado el contexto de diversidad sociolingüística, donde las personas podrían recibir información en su idioma, de una forma cercana y considerando su realidad local.

Idealmente el sistema de vigilancia debe centrarse en los intereses de los pueblos, con tres subsistemas de monitoreo crítico, reacción inmediata y participación comunicativa con carácter participativo ${ }^{7}$ y con vigilancia sociocultural de la salud ${ }^{69}$. Sin embargo, en Guatemala el ejercicio del biopoder por medio de la utilización de fuerzas militares ha sido una constante, con creación de cordones sanitarios por personal militar y policial para garantizar la permanencia de las personas en sus casas, durante los primeros casos por Covid-19.

Aunque parece imposible revertir años de desfinanciamiento y del modelo de atención en plena crisis en países como Guatemala, quizás sería importante tomar lecciones de países como Sierra Leona que aún con epidemia de Ébola lograron reducir la pobreza multidimensional fortaleciendo su institucionalidad pública ${ }^{70}$.

\section{Promoción e intersectorialidad en salud}

El papel de un sistem a basado en APS exige nuevas estrategias de gobernanza para garantizar cambios en la gestión institucional y en el acompañamiento de resultados, donde las comunidades desempeñan un papel fundamental, tanto en la elaboración como en la implementación de políticas, fomentando el empoderamiento de sujetos individuales y colectivos en la búsqueda de la equidad71. El enfoque de género es fundamental, de hecho en situaciones de crisis humanitarias las mujeres son las que han creado mejores planes para la contención de consecuencias ${ }^{\mathbf{7 2}}$.

La transparencia y el acceso a la información pública son un reto por superarse buscando mecanismos de institucionalización de estas prácticas, más allá de la existencia de arreglos institucionales como, por ejemplo, una ley específica, sino por medio de un compromiso social y cultural73 de la ciudadanía en una lucha constante. Una clave importante para afrontar la pandemia ha sido precisamente una política de comunicación clara, efectiva, coherente y enfocada a eliminar las informaciones falsas ${ }^{74}$, ya que comunicaciones descoordinadas o contradictorias comprometen la respuesta de implementación de políticas y la confianza 
de la población hacia las políticas oficiales ${ }^{75}$.

El enfoque de promoción de la salud es fundamental como un lugar analítico y metodológico, donde el abordaje de los problemas de salud no se restringe al uso de tecnología médica, por el contrario, es una práctica social que varía de acuerdo al contexto y debe abordar las diferentes inequidades, superando algunas limitaciones como el predomino del pensamiento occidental (presente en la declaración de Ottawa), y la confusión existente entre promoción y prevención ${ }^{76,77}$.

En Guatemala, el papel de la sociedad civil organizada ha sido importante, pero deslegitimado por el gobierno. Los programas de protección social fueron implementados de forma centralizada sin mecanismos de coordinación o consulta con, por ejemplo, pueblos indígenas, mujeres, población en condición de vulnerabilidad, diversidad sexual, autoridades locales o municipales y otras. Las decisiones de restricción de movilidady de las actividades económicas esenciales han sido modificadas conforme críticas del sector privado organizado 9 . Además, sin enfoque de género, el discurso oficial ha sido predominantemente masculino ${ }^{44}$.

Esto ha provocado que la implementación de los programas sociales no sea adecuada. Más de la mitad de las familias en situación de pobreza no ha recibido ningún tipo de beneficio, la forma de inscripción es engorrosa, con medios digitales para su inscripción y sin posibilidad de alcance a familias analfabetas y sin recursos ${ }^{9,78}$. No existen datos desagregados por género, condición de discapacidad u otros. Existe baja capacidad de ejecución y falta de criterios técnicos para la coordinación interministerial que requiere su operativización. Por ejemplo, el programa de alimentación posee una ejecución de 0 y del adulto mayor 5.18\%, al mes de julio de $2020^{79}$.

Como parte de los comités consultivos para tomar decisiones, fue creada en mayo, dos meses después de la declaración de la pandemia, la comisión presidencial para la atención de la emergencia, en la cual participan diferentes ministerios, la secretaria de planificación de la presidencia y un director ejecutivo cuyo perfil es de médico epidemiólogo ${ }^{80}$. Al no contar con una estrategia de comunicación definida, tanto el comisionado, como el presidente y el ministerio, realizan declaraciones, en algunos casos contradictorias y de forma confusa. Además, la estrategia de comunicación no ha sido adaptada culturalmente.

Según declaraciones oficiales se ha dicho que el mejor camino es la reapertura de la economía y que será necesario acostumbrarse a vivir con el virus, bajo la nueva normalidad, lo que no es más que continuar las exigencias del neoliberalismo, invisibilizando las inequidades, la precarización del trabajo y sobre todo la pérdida de vidas humanas.

Esto indica que además de formas negacionistas, también es posible ejercer el poder para racionalizar la economía y la sociedad defendiendo los grandes intereses corporativos y las necesidades estratégicas del capital, asumiendo como propio el positivismo como ciencia, y rechazando toda forma de ciencia crítica $^{81}$. El papel de la comisión presidencial muestra que inclusive académicos viven engañados al pensar que por tener fines legítimos pueden realizar una epidemiología liberadora, centrando sus denuncias, por medio de modelos matemáticos con alta precisión, en los fenómenos superficiales ${ }^{7(38)}$ sin abordar las causas estructurales asociadas a los patrones de enfermedad, atención y desatención.

Esa forma de organización supone crear estrategias de responsabilización individual, transformando cada trabajador en un sujeto responsable por sus propios éxitos y fracasos, ignorando su género, su contexto social, cultural, de formación y de existencia, donde la lógica es que el mercado no puede parar y debe funcionar a pesar de todo, de mascarilla y lentes de protección, en una especie de necropolítica ${ }^{\mathbf{8 1}}$.

La falta del acceso a la información sobre el número de casos, número de pruebas realizadas y bases de datos para realizar análisis independientes son una limitante para el ejercicio pleno de una ciudadanía crítica. Datos 
oficiales son divulgados a través del formato de infografía y al intentar realizar búsquedas en bases de datos completas $\mathbf{8 2}$ para realizar comparaciones, proyecciones, análisis de perfil o cualquier otro tipo de análisis entre países no existen datos actualizados.

En medio de la crisis sanitaria existe una crisis política entre la destitución de autoridades ministeriales tras escándalos de corrupción y falta de transparencia de los datos. La coordinación entre el poder ejecutivo, legislativo y judicial no ha sido positiva y se ha entrecruzado con elección de magistraturas, intentos de negar información y pedir la destitución del Procurador de los Derechos Humanos y de otras instancias de la sociedad civil, como autoridades mayas y jóvenes indígenas, quienes han exigido respuestas ante las demandas de la población.

La baja capacidad de articulación con la población, aunque agudizada en este gobierno, no es sorpresa en una democracia categorizada como híbrida. De hecho, es el sexto país con el índice de democracia más bajo de América Latina y ocupa el puesto 93 a nivel global83. Mientras tanto la comunidad internacional y las comunidades locales, con las mujeres y grupos de jóvenes en redes de solidaridad, han creado programas de asistencia ante la falta de presencia del Estado.

\section{Consideraciones finales}

La APS posee un papel fundamental para abordar crisis sanitarias como la pandemia, y para garantizar el cuidado de la población adscrita y la vigilancia en salud. Esto debe ser motivo de redireccionamiento de las acciones actuales, están centradas únicamente en el nivel hospitalario y su fortalecimiento a largo plazo, no sólo para atender el coronavirus sino como abordaje efectivo de retos futuros.

En Guatemala, la inequidad que deja en situación de vulnerabilidad a comunidades mayas, mujeres, indígenas, migrantes y disidencias sexuales, durante este periodo ha visto incrementada la pobreza, desnutrición y la falta de acceso a servicios de salud pública, vivienda y saneamiento básico. La baja ejecución del presupuesto de salud, la creación de hospitales de campaña con donaciones y la aprobación de seguros privados alejan la idea del fortalecimiento del sistema salud y de la atención primaria.

El potencial de la APS para la participación social e intersectorialidad ha sido limitado en el país guatemalteco. La respuesta ha sido centralizada y con medidas autoritarias del gobierno, inclusive con la utilización de fuerzas armadas, sin considerar el papel de la sociedad civil en los canales de diálogo para la co-construcción en la formulación de políticas públicas y rendición de cuentas que promuevan canales abiertos, fortalecimiento de la ciudadanía y de las múltiples organizaciones sociales y ancestrales.

Tanto la experiencia internacional como la regional demuestran que la APS sigue siendo importante, a pesar del abordaje mediático a mecanismos de alta complejidad, tanto para la coordinación del cuidado, la vigilancia sanitaria y el reconocimiento de la realidad local, como para abordar las vulnerabilidades sociales y para promover mecanismos de participación social para la transformación de la determinación social de la salud.

En el contexto guatemalteco es necesario pensar que una transformación y fortalecimiento de salud no pueden aislarse del fortalecimiento democrático y de la protección social, considerando la equidad de género, la pertinencia intercultural, y un enfoque territorial y de gestión del cuidado con intervenciones familiares y comunitarias. Esto lleva a pensar en el propio papel del Estado e inclusive en una reforma sanitaria basada en la APS con aumento expresivo del acceso, financiamiento, incidencia en la calidad de vida con dignidad de sus habitantes, y formación del talento humano; todo esto bajo la óptica de la integralidad y equidad como ejes transversales para la garantía de la salud como derecho universal. 


\section{Agradecimientos}

Agradezco a la Profa. Dra. Ana Luiza Vilasbôas por la lectura crítica del manuscrito.

\section{Colaborador}

Figueroa CDO (0000-0002-6278-8265)* es el encargado de preparar el manuscrito.

\section{Referencias}

1. Machhi J, Herskovitz J, Senan AM, et al. The Natural History, Pathobiology, and Clinical Manifestations of SARS-CoV-2 Infections. J Neuroimmune Pharmacol. 2020; 15(3):359-386.

2. Organização Pan-americana da Saúde Brasil, Organização Mundial da Saúde. Folha informativa COVID-19 (doença causada pelo novo coronavírus). [acceso en 2020 ago 15]. Disponible en: https://www.paho.org/ bra/index.php?option=com $\_$content\&view=article\&i$\mathrm{d}=6101$ :covid19\&Itemid $=875$.

3. Aquino EML, Silveira IH, Pescarini JM, et al. Medidas de distanciamento social no controle da pandemia de COVID-19: potenciais impactos e desafios no Brasil. Ciênc. Saúde Colet. 2020; 25(supl):2423-2446.

4. Peña S, Cuadrado C, Rivera-Aguirre A, et al. PoliMap: A taxonomy proposal for mapping and understanding the global policy response to COVID-19. [Preprint]. [ acceso en 2020 abr 2]. Disponible en: doi:10.31219/ osf.io/h6mvs.

5. Gondim R. Atenção Primária à Saúde e populações vulnerabilizadas: potencialidades e contradições da atenção à saúde de pessoas em situação de rua. In: Mendonça MHM, Matta GC, Gondim R, et al., organizadores. Atenção primária à saúde no Brasil: conceitos, práticas e pesquisa. Rio de Janeiro: SciELO-Editora; FIOCRUZ; 2018. p. 197-230.
6. Medina MG, Vilasbôas AL, Nunes C, et al. Atenção primária à saúde: reflexões sobre a política a partir da prática de pesquisa. In: Teixeira CF, organizador. Observatório em análise política em saúde: abordagens, objetos e investigações. Salvador: EDUFBA; 2016. p. 227-265.

7. Breilh J. SARS-CoV2: rompiendo el cerco de la ciencia del poder. Escenario de asedio de la vida, los pueblos y la ciencia. In: Amadeo P, organizador. Posnormales: pensamiento contemporáneo en tiempos de pandemia. La Plata: ASPO; 2020. p. 31-90.

8. Organización Panamericana de la Salud, Organización Mundial de la Salud. La renovación de la atención primaria de salud en las Américas: Documento de Posición de la Organización Panamericana de la Salud/Organización Mundial de la Salud. Washington, DC: OMS; OPAS; 2007.

9. Shadmi E, Chen Y, Dourado I, et al. Health equity and COVID-19: global perspectives. Int J Equity Health. 2020; 19(1).

10. Noronha KVMS, Guedes GR, Turra CM, et al. Pandemia por COVID-19 no Brasil: análise da demanda e da oferta de leitos hospitalares e equipamentos de ventilação assistida segundo diferentes cenários. Cad. Saúde Pública. 2020; 36(6):1-17.
${ }^{*}$ Orcid (Open Researcher and Contributor ID). 
11. Medina MG, Giovanella L, Bousquat A, et al. Atenção primária à saúde em tempos de COVID-19: o que fazer? Cad. Saúde Pública. 2020; 36(8):1-5.

12. Brasil. Secretaria da Saúde do Estado da Bahia. Organização da APS pelo mundo durante a pandemia da COVID-19. Salvador: SSEB; 2020.

13. Instituto Nacional de Estadística. Resultados del XII Censo Nacional de Población y VII de Vivienda. Guatemala: INER; 2019.

14. Guatemala. Ministerio de Desarrollo Social. Iniciativa sobre la Pobreza y Desarrollo Humano de Oxford (OPHI). IPMGt: Índice de pobreza multidimensional. Guatemala: MD; 2018.

15. Comisión Económica para América Latina y el Caribe. Panorama Social de América Latina, 2019. Santiago: CEPAL; 2019.

16. Programa das Nações Unidas para o Desenvolvimento. Progreso multidimensional: bienestar más allá del ingreso. New York: PNDU; 2016.

17. Organización Internacional del Trabajo. La economía informal en Centroamérica y República Dominicana: desarrollo subregional y estudios de caso. San José: Organización Internacional del Trabajo; 2013.

18. World Bank Group. World Development Indicators database. 2020. [acceso en 2020 jun 6]. Disponible en: http://datatopics.worldbank.org/world-development-indicators/.

19. Organização das Nações Unidas. Levels and Trends in Child Mortality. Geneva: ONU; 2019.

20. Guatemala. Ministerio de Salud Pública y Asistencia Social. Informe de país. Situación de la mortalidad materna 2014-2015. Guatemala: MSP; 2017.

21. Food and Agriculture Organization of the United Nations; Organização Pan-Americana da Saúde; World Food Programme; United Nations International Children's Emergency Fund. Panorama de la seguridad alimentaria y nutricional en América Latina y el Cari- be 2019. Santiago: FAO; OPAS; WFP; UNICEF; 2019. [acceso en 2020 mar 25]. Disponible en: http://www. fao.org/publications/es.

22. Food and Agriculture Organization of the United Nations. Panorama de la seguridad alimentaria y nutricional en Centroamérica y República Dominicana. Ciudad de Panamá: FAO; 2015.

23. Programa das Nações Unidas para o Desenvolvimento. Informe sobre Desarrollo Humano 2019. Más allá del ingreso, más allá de los promedios, más allá del presente: Desigualdades del desarrollo humano en el siglo XXI. New York: PNUD; 2019.

24. Becerrill V, López L. Sistema de salud de Guatemala. Salud Publica Mex. 2011 [acceso en 2015 sep 18]; 53(2):s197-s208. Disponible en: http://www. scielo.org.mx/scielo.php?script=sci_arttext\&pi$\mathrm{d}=$ S0036-36342011000800015\&lng=es\&nrm=iso\&tlng=es.

25. Instituto de Salud Incluyente. El camino a la transformación del sistema público de salud: plan maestro nacional de la red de atención en salud, completando la espiral. Guatemala: Serviprensa S.A.; 2019.

26. Cerón A. Los caminos del enfermo en una comunidad K'iche' de Guatemala: una contribución del análisis de redes al estudio de los comportamientos de búsqueda de atención en salud. Rev Hisp para el análisis redes Soc. 2010 [acceso em 2020 oct 10]; 18(1). Disponible en: https://digitalcommons.du.edu/anthropology_faculty/13.

27. Instituto Guatemalteco de Seguridad Social. Plan estratégico institucional 2018-2022. Guatemala: IGS; 2017.

28. Verdugo JC. La política y reforma del Sector Salud en Guatemala 1986-2000. In: Avila C, Bright R, Gutiérrez JC, et al., organizadores. Análisis sobre la Reforma del Sector Salud en Guatemala. Guatemala: USAID: HFG; 2005. p. 9-36.

29. Verdugo JC. The failures of neoliberalism: Health sector reform in Guatemala. In: Fort M, Mercer MA, 
Gish O, organizadores. Sickness and Wealth: The Corporate Assault on Global Health. Massachusetts: South End Press; 2004. p. 57-68.

30. Universidad Rafael Landívar; Organización Panamericana de la Salud. Información sobre recursos humanos de salud en Guatemala. Guatemala: Universidad Rafael Landívar; 2009. [acceso en 2020 abr 1]. Disponible en: https://www.paho.org/hq/dmdocuments/2010/RH_GUA_Informe_RRHH_enero_2010. pdf.

31. Guatemala. Ministerio de Salud Pública y Asistencia Social. Cuentas Nacionales de Salud. Financiamiento de la salud en Guatemala período 1995-2014. Guatemala: MSPAS; 2015.

32. Guatemala. Ministerio de Salud Pública y Asistencia Social. Financiamiento y gasto en salud. Guatemala periodo 2016-2017. Guatemala: Ministério de Salud Pública y Asistência Social; 2016.

33. Guatemala. Ministerio de Salud Pública y Asistencia Social; Universidad de San Carlos de Guatemala. Evaluación de Programas de Recursos Humanos en Salud. Guatemala; 2013. [acceso en 2020 ago 11]. Disponible en: https://www.observatoriorh.org/sites/default/files/webfiles/fulltext/2013/evalprogra instrum4_gua.pdf.

34. Guatemala. Ministerio de Salud Pública y Asistencia Social. Segundo año de gobierno: memoria de labores 2017-2018. Guatemala: MSPAS; 2018.

35. Guatemala. Ministerio de Salud Pública y Asistencia Social. Modelo de atención y gestión para áreas de salud. Guatemala: MSPAS; 2018.

36. Berger-González M, Stauffacher M, Zinsstag J, et al. Transdisciplinary Research on Cancer-Healing Systems Between Biomedicine and the Maya of Guatemala. Qual Health Res. 2016; 26(1):77-91.

37. Consejo Mayor de Médicos Maya’ob' por Nacimiento; Berger-González M, organizador. Raxnaq'il Nuk'aslemal: Medicina Maya'en Guatemala. Guatemala: Iximulew; 2016.
38. Nómada. (Liveblog): Ahora que llegamos a 176 casos en un día, Giammattei endurece medidas. Noticias y actualizaciones sobre la emergencia sanitaria relacionada al coronavirus (Covid-19) y las medidas de prevención en Guatemala. 2020 mayo 14 [acceso em 2020 jul 20]. Disponível en: https://nomada.gt/ pais/actualidad/liveblog-el-coronavirus-y-la-emergencia-sanitaria/.

39. Comisión Económica para América Latina y el Caribe. Organización Panamericana de la Salud. Informe CEPAL-OPS Salud y economía: una convergencia necesaria para enfrentar el COVID-19 y retomar la senda hacia el desarrollo sostenible en América Latina y el Caribe. [acceso em 2020 ago 11]. Disponible en: https://repositorio.cepal.org//handle/11362/45840.

40. Pérez J. Decretos oficializan (pero no aclaran) quién recibirá la ayuda por la crisis del COVID19 | Plaza Pública. Plaza Pública. [acceso em 2020 ago 11]. Disponible en: https://www.plazapublica.com.gt/content/ decretos-oficializan-pero-no-aclaran-quien-recibira-la-ayuda-por-la-crisis-del-covid-19.

41. OCHA Services. Guatenala COVID-19: Informe de situación No. 05. [acceso en 2020 jul 20]. Disponible en: https://reliefweb.int/report/guatemala/guatemala-covid-19-informe-de-situaci-n-no-05-al-08de-junio-2020.

42. OCHA Services. Guatemala: COVID-19 Informe de Situación No. 04. [acceso en 2020 jul 20]. Disponible en: https://reliefweb.int/report/guatemala/guatemala-covid-19-informe-de-situaci-n-no-04-al-30de-abril-2020.

43. Matamoros W. Realidades de las mujeres trans: "los derechos humanos no pueden ser puestos en cuarentena". Fondo Centroamericano de Mujeres. [acceso en 2020 ago 12]; Disponible en: https://www.fcmujeres.org/es/ realidades-de-las-mujeres-trans-los-derechos-humanos-no-pueden-ser-puestos-en-cuarentena/.

44. Monzón AS, Peruch K. Mujeres, cuidados y violencia: consecuencias del confinamiento por la pandemia del COVID-19. Quetzaltenango: Mujeres Transformando al Mundo; 2020. 
45. Observatorio en Salud Reproductiva. Monitoreo de embarazos en adolescentes entre 10 y 19 años al 20 de mayo 2020. Información y datos. [acceso en 2020 jul 10]. Disponible en: https://osarguatemala.org/monitoreo-de-embarazos-en-adolescentes-entre-10-y-19anos-al-20-de-mayo-2020/.

46. Flores P. Parque de la Industria sin oxígeno suficiente: fallece joven de 22 años. Nómada Guatemala. [acceso em 2020 ago 15]. Disponible en: https://nomada. gt/pais/actualidad/liveblog-parque-de-la-industriadespues-de-79-dias-sin-salarios-llegan-las-primerassoluciones/.

47. Guatemala. Ministerio de Educación. Acuerdo Gubernativo 44-2020: Crear el programa seguro médico escolar. Guatemala: Diario de Centroamérica; 2020.

48. Guatemala. Ministerio de Salud Pública y Asistencia Social. El MSPAS entregará kit de medicamentos COVID-19 para pacientes con síntomas leves. Ministerio de Salud Pública y Asistencia Social. [acceso en 2020 jul 19]. Disponible en: https://mspas.gob.gt/index. $\mathrm{php} /$ noticias/noticias-mspas/item/933-el-mspas-entregara-kit-de-medicamentos-covid-19-para-pacientes-con-sintomas-leves.

49. Nómada. Resumen del día: Esto incluyen los kits, de las munis de Guatemala y Mixco, contra el COVID-19. Nómada. [acceso em 2020 jul 19]. Disponible en: https://nomada.gt/pais/actualidad/resumen-del-diaesto-incluyen-los-kits-de-las-munis-de-guatemala-y-mixco-contra-el-covid-19/.

50. Hernández Mack L. Análisis de ejecución presupuestaria, adquisiciones y abastecimiento del Ministerio de Salud Pública y Asistencia Social durante la emergencia del COVID-19. Guatemala: MASPAS; 2020.

51. Melo E, Mattos R. Gestão do cuidado e atenção básica: controle ou defesa da vida? In: Mendoça $\mathrm{MH}$, Matta GC, Gondim R, et al., organizadores. Atenção primária à saúde no Brasil: conceitos, práticas e pesquisa. Rio de Janeiro: SciELO-Editora; FIOCRUZ; 2018. p. 95-116.
52. Organisation for Economic Co-operation and Development. Realising the Potential of Primary Health Care. Paris: OECD Publishing; 2020.

53. Organização Pan-Americana da Saúde. Reforçando a resposta dos sistemas de saúde à COVID-19. Adaptando serviços de atenção primária para uma resposta mais efetiva à COVID-19. Brasilía, DF: Organização Pan-Americana da Saúde; 2020.

54. Kidd MR. Five principles for pandemic preparedness: Lessons from the Australian COVID-19 primary care response. Br J Gen Pract. 2020; 70(696):316-317.

55. Majeed A, Maile EJ, Bindman AB. The primary care response to COVID-19 in England's National Health Service. J R Soc Med. 2020; 113(6):208-210.

56. Gondim GMM, Monken M. O uso do território na Atenção Primária à Saúde. In: Mendoça MH, Matta GC, Gondim R, et al., organizadores. Atenção primária à saúde no Brasil: conceitos, práticas e pesquisa. Rio de Janeiro: SciELO-Editora FIOCRUZ; 2018. p. 143-156.

57. Santos AL, Rigotto RM. Território e territorialização: incorporando as relações produção, trabalho, ambiente e saúde na atenção básica à saúde. Trab Educ e Saúde. 2010;8(3):387-406.

58. Junior AGS, Latgé PK, Oliveira RAT, et al. A experiência de Niterói no enfrentamento da COVID 19: notas preliminares sobre a articulação de políticas sociais e de saúde. APS EM Rev. 2020; 2(2):128-136.

59. Gurung G, Atmore C, Gauld R, et al. Integrated ambulatory care in the New Zealand health system: a scoping review. J Integr Care. 2020; 28(3):253-280.

60. Giovanella L, Stegmüller K. The financial crisis and health care systems in Europe: Universal care under threat? Trends in health sector reforms in Germany, the United Kingdom, and Spain. Cad. Saúde Pública. 2014; 30(11):2263-2281. 
61. Armocida B, Formenti B, Ussai S, et al. The Italian health system and the COVID-19 challenge. The Lancet. 2020; 5(5):e253.

62. Cartabellotta N, Cottafava E, Luceri R, et al. Report Osservatorio GIMBE n. 7/2019. Il definanziamento 2010-2019 del Servizio Sanitario Nazionale. [acceso en 2020 ago 10]. Disponible en: https://www. gimbe.org/osservatorio/Report_Osservatorio_GIMBE_2019.07_Definanziamento_SSN.pdf.

63. García-Basteiro A, Alvarez-Dardet C, Arenas A, et al. The need for an independent evaluation of the COVID-19 response in Spain. Lancet (London). 2020; 396(10250):529-530.

64. Leal Souza MSP, Aquino R, Pereira SM, et al. Fatores associados ao acceso geográfico aos serviços de saúde por pessoas com tuberculose em três capitais do Nordeste brasileiro. Cad. Saúde Puública. 2015; 31(1):111-120.

65. Instituto Nacional de Estadística. Encuesta Nacional de Condiciones de Vida (ENCOVI): 2014. [acceso en 2020 ago 11]. Disponible en: https://www.ine. gob.gt/sistema/uploads/2016/02/03/bWC7f6t7aSbEI4wmuExoNROoScpSHKyB.pdf.

66. World Health Organization. Role of primary care in the COVID-19 response. Western Pacific Region: WHO; 2020.

67. Nómada. Declaraciones de Edwin Asturias. [acceso en 2020 ago 15]. Disponible en: https://www.facebook.com/watch/?v=304677657559915\&extid=hbNcYC8SHIrFOO2r.

68. Teixeira MG, Medina MG, Costa MCN, et al. Reorganization of primary health care for universal surveillance and containment of COVID-19. Epidemiol e Serv saude Rev do Sist Unico Saude do Bras. 2020; 29(4):e2020494.

69. Hersch-Martínez P. Epidemiología sociocultural: una perspectiva necesaria. Salud Publica Mex. 2013 [acceso en 2020 ago 11]; 55(5):512-518. Disponible en: http://www.scielo.org.mx/scielo.php?script=sci_arttext\&pid=S0036-36342013000700009\&lng=es\&nr$\mathrm{m}=$ iso.

70. Oxford Poverty and Human Development Initiative; United Nations Development Programme. Charting pathways out of multidimensional poverty: achieving the SDGs. [acceso en 2020 ago 15]. Disponible en: http://hdr.undp.org/en/content/2020-global-multidimensional-poverty-index-mpi.

71. Tavares MFL, Rosana RM, Magalhães R. Promoção da saúde e o desafio da intersetorialidade na Atenção Básica no Brasil. In: Mendonça MHM, Matta GC, Gondim R, et al., organizadores. Atenção primária à saúde no Brasil: conceitos, práticas e pesquisa. Rio de Janeiro: SciELO-Editora FIOCRUZ; 2018. p. 177196.

72. Saavedra AGF, Moreno SD. La integración de la perspectiva de género en la gestión del riesgo de desastres: de los ODM a los ODS. Rev Int Coop y Desarro. 2018; 5(1):31-43.

73. Costa JM. La construcción de ciudadanía desde el derecho de acceso a la información pública. Rev la Fac Derecho y Ciencias Soc. 2019 [acceso en 2020 ago 12]; (2019):157-176. Disponible en: http://www.der.una. py/application/files/9415/9008/4735/20190521-revista-academica-facultad-de-derecho-una-2019.pd$\mathrm{f} \#$ page $=158$.

74. Ratzan SC, Sommariva S, Rauh L. Enhancing global health communication during a crisis: lessons from the COVID-19 pandemic. Public Heal Res Pract. 2020; $30(2)$.

75. Ratzan SC, Gostin LO, Meshkati N, et al. COVID-19: An Urgent Call for Coordinated, Trusted Sources to Tell Everyone What They Need to Know and Do. NAM Perspect. 2020 mar.

76. McPhail-Bell K, Fredericks B, Brough M. Beyond the accolades: a postcolonial critique of the foundations of the Ottawa Charter. Glob Health Promot. 2013; 20(2):22-29. 
77. Heidmann ITSB, Almeida MCP, Boehs AE, et al. Promoção à saúde: trajetória histórica de suas concepções. Texto Context - Enferm. 2006; 15(2):352358.

78. Oxfam. Paraíso Desigual, Pacto Ciudadano. Primer informe: monitoreo participativo del bono familia. Guatemala: Oxfam; 2020.

79. Guatemala. Comisión Presidencial COVID-19. Informe mensual al Congreso de la República. [acceso en 2020 abr 1]. Disponible en: https://osarguatemala. org/wp-content/uploads/2020/07/Presentacio\%CC\%8ln-COPRECOVID-Informe-Mensual-Congreso. pdf.

80. Guatemala. Acuerdo gubernativo 65-2020: creación de la Comisión Presidencial de Atención a la Emergencia COVID-19 (Coprecovid). Guatemala: Diario de Centroamérica; 2020.
81. Caponi S. Covid-19 no Brasil: entre o negacionismo e a razão neoliberal. Estud Avançados. 2020; 34(99):209224.

82. Roser M, Ritchie H, Ortiz-Ospina E, et al. Coronavirus Pandemic (COVID-19). Our World Data. [acceso en 2020 ago 10]. Disponible en: https://ourworldindata.org/coronavirus.

83. The Economist Intelligence Unit. Democracy Index 2019: A year of democratic setbacks and popular protest. London: EUI; 2020. [acceso en 2020 jul 19]. Disponible en: http://www.eiu.com/Handlers/WhitepaperHandler.ashx?fi=Democracy-Index-2019.pd$\mathrm{f} \&$ mode $=w p \& c a m p a i g n i d=$ democracyindex 2019 .

Recibido en 20/08/2020

Aprobado en 08/06/2021

Conflicto de intereses: inexistente

Apoyo financiero: Consejo Nacional de Desarrollo Científico y

Tecnológico (CNPq). Código de proceso (141960 / 2020-5) 